\title{
A new mean-field plasma edge transport model based on turbulent kinetic energy and enstrophy
}

\author{
R. Coosemans, W. Dekeyser, M. Baelmans
}

Applied Mechanics and Energy Conversion Section, KU Leuven, Department of Mechanical Engineering,

Celestijnenlaan 300 box 2421, 3001 Leuven, Belgium

reinart.coosemans@kuleuven.be

Accepted on 18 December 2019

This is the peer reviewed version of the following article: $R$. Coosemans, W. Dekeyser, M. Baelmans. A new mean-field plasma edge transport model based on turbulent kinetic energy and enstrophy. Contributions to Plasma Physics. 2020;e201900156., which has been published in final form at https://doi.org/10.1002/ctpp.201900156. This article may be used for non-commercial purposes in accordance with Wiley Terms and Conditions for Use of Self-Archived Versions.

\begin{abstract}
The predictive capabilities of mean-field plasma edge transport codes are significantly reduced by the empirically determined radial diffusion coefficients commonly used to account for the turbulent transport. This contribution aims to assess a two-equation model including both turbulent kinetic energy and turbulent enstrophy for an improved description of the mean-field turbulent transport. A turbulent enstrophy equation is analytically derived for isothermal, electrostatic, 2D, interchange-dominated ExB turbulence in the scrape-off layer. Evaluation of this equation shows that interchange is the dominant source, while the sheath loss and viscous terms provide the main sinks of turbulent enstrophy. Transport effects turn out to be of minor importance. A model for the combined turbulent kinetic energy and enstrophy equations and the particle transport is constructed starting from a one-equation model for the turbulent kinetic energy. The resulting two-equation model is shown to retain the dominant dynamics of the latter, while improving its scalings.
\end{abstract}

Key words: SOL, ExB turbulence, turbulent kinetic energy, turbulent enstrophy 


\section{Introduction}

Transport processes in the plasma edge of magnetically confined fusion reactors are generally known to be governed by ExB drift turbulence $[1,2,3]$, but $3 \mathrm{D}$ turbulence simulations of reactor-scale machines are still beyond the existing computational capabilities. Hence, mean-field plasma edge transport codes remain key tools for the design of nuclear fusion reactors and the divertor in particular. However, the predictive capabilities of these codes are significantly reduced by ad-hoc, empirically determined radial diffusion coefficients used to account for the turbulent transport $[4,5,6]$. To improve the transport description, Bufferand et al. [7] and Coosemans et al.[8] presented models that relate this diffusion coefficient to the turbulent kinetic energy $\left(k_{\perp}\right)$, together with mean-field transport equations for the turbulent kinetic energy. The resulting transport models contain only a few model parameters. Baschetti et al.[9] used machine scaling laws to obtain a closure for the $k_{\perp}$ equation proposed by Bufferand et al., while Coosemans et al. used reference data from a turbulence code to model the $k_{\perp}$ equation.

While the model proposed by Coosemans et al. managed to fit turbulence reference data rather well over a range of parameters, it was unable to capture some trends, especially those with varying viscosity. Moreover, the model lumped the different sinks of the turbulent kinetic energy together in one contribution. In this work we aim to extend this model by including an equation for the turbulent enstrophy $\left(\zeta_{\perp}\right)$, as there are convincing physical grounds to expect the enstrophy to provide additional information about the turbulent transport in the plasma edge.

The remainder of this paper is structured as follows. Section 2 introduces the TOKAM2D turbulence code $[10,11,12]$ that is used as a reference in this work, as well as the one-equation $k_{\perp}$ model. Next, section 3 defines the enstrophy, and shows that the turbulent enstrophy $\left(\zeta_{\perp}\right)$ can be combined with the turbulent kinetic energy to obtain an improved model for the turbulent particle diffusion coefficient. A turbulent enstrophy equation is derived analytically for the TOKAM2D model. It is used to evaluate the enstrophy balance using TOKAM2D reference data. Section 4 then develops a closed transport model for the combined $k_{\perp}-\zeta_{\perp}$ system and the average particle transport. Section 5 shows results obtained with the new model and compares them to the one-equation $k_{\perp}$ model. Finally, section 6 summarizes the main findings and results of this work.

\section{TOKAM2D and turbulent kinetic en- ergy equations}

In this paper, isothermal, electrostatic, 2D, interchange-dominated ExB turbulence in the scrape-off layer (SOL) is considered. Under these as- 
sumptions, the equation set reduces to $[10,11,12]$

$$
\begin{gathered}
\frac{\partial n}{\partial t}+[\phi, n]=S_{n}-\sigma_{N} c_{s} n \exp \left(\Lambda-\frac{\phi}{T_{e}}\right)+D_{n} \nabla_{\perp}^{2} n, \\
\frac{\partial \omega}{\partial t}+[\phi, \omega]=\frac{1}{n}[n T, g x]+\sigma_{W} c_{s}\left(1-\exp \left(\Lambda-\frac{\phi}{T_{e}}\right)\right)+\nu \nabla_{\perp}^{2} \omega, \\
\omega=\nabla_{\perp}^{2} \phi .
\end{gathered}
$$

In these equations, $n$ is the density, $\phi$ the electrostatic potential, $\omega$ the vorticity, $S_{n}$ a particle source, $\sigma$ a parameter characterizing the losses to the sheath, $c_{s}$ the sound speed, $T$ the temperature, $\Lambda$ the sheath potential, $D_{n}$ a diffusion coefficient, $g$ a parameter characterizing the magnetic field curvature (see for example Ref. 10 for an analytical expression) and $\nu$ a viscosity. The Poisson bracket is defined as $[P, Q]=b \cdot(\nabla P \times \nabla Q)$, where $b$ is the magnetic field unit vector. Note that all quantities in these equations are normalized to the reference ion gyro-frequency $\Omega_{r e f}=q B_{r e f} / \mathrm{m}$ and gyro-radius $\rho_{\text {ref }}=\Omega_{r e f}^{-1} \sqrt{T_{r e f} / m}$, where $q$ is the ion charge, $m$ the ion mass and $B_{\text {ref }}$ the magnetic field strength. All equations in the remainder of this paper will follow this same normalisation. Reference data for the development of mean-field models for the turbulent transport is provided by the finite volumes version of the TOKAM2D turbulence code $[11,12]$, which is run in isothermal mode such that $T_{i}$ and $T_{e}$ are considered to be fixed parameters. Hence, this code solves equations 1 to 3 on a fine 2D mesh with a small time step in order to resolve all the time and length scales of the turbulence. The $2 \mathrm{D}$ mesh includes the radial direction of the tokamak and the diamagnetic direction, which is a periodic direction. Hence, average quantities of the plasma only vary in the radial direction as the parallel direction is not resolved but replaced by a model for the sheath.

Starting from this TOKAM2D turbulence model, the corresponding $k_{\perp}$ equation can be derived analytically [8]:

$$
\begin{array}{r}
\frac{\partial}{\partial t} \bar{n} k_{\perp}+\nabla \cdot\left(\bar{\Gamma}_{k_{\perp}}\right)+\frac{\sigma_{N} c_{s}}{2} \overline{n V_{E}^{\prime \prime 2} \exp \left(\Lambda-\frac{\phi}{T_{e}}\right)} \\
=\underbrace{-\overline{\phi^{\prime}([n T, g x])^{\prime}}}_{\text {interchange }}-\underbrace{\sigma_{W} \overline{\phi^{\prime}\left(c_{s} n\left(1-\exp \left(\Lambda-\frac{\phi}{T_{e}}\right)\right)^{\prime}\right.}}_{\text {sheath }{ }_{\text {loss }}}-\underbrace{\nu \overline{\phi^{\prime}\left(n \nabla_{\perp}^{2} \omega\right)^{\prime}}}_{\text {viscosity }} \\
-\overline{n V_{E}^{\prime \prime} V_{E}^{\prime \prime}}:\left(\nabla \tilde{V}_{E}\right)^{T}+\frac{\bar{J}_{p}}{\bar{n}} \overline{n^{\prime} \nabla \phi^{\prime}}+\overline{\phi^{\prime}\left(V_{p} \cdot \nabla n\right)^{\prime}}+\frac{1}{2} \overline{V_{E}^{\prime \prime 2} S_{n}}+\frac{D_{n}}{2} \overline{V_{E}^{\prime \prime 2} \nabla_{\perp}^{2} n}, \\
\bar{\Gamma}_{k_{\perp}}=\bar{n} \tilde{V}_{E} k_{\perp}+\overline{n V_{E}^{\prime \prime} V_{E}^{\prime \prime 2}} / 2+\overline{\phi^{\prime} J_{p}^{\prime}} .
\end{array}
$$

In these equations, Reynolds averages are denoted by an overbar $(\bar{x}=$ $\left.\lim _{T \rightarrow \infty} \int_{0}^{T} x d t\right)$ and Favre averages by a tilde $(\tilde{x}=\overline{n x} / \bar{n})$ such that turbulent quantities can be split in mean-flow and fluctuating components (as $x=\bar{x}+x^{\prime}$ or $x=\tilde{x}+x^{\prime \prime}$ respectively) $[13,14] . V_{E}$ is the ExB drift velocity, $J_{p}$ the polarization current and the turbulent kinetic energy is defined as $\bar{n} k_{\perp}=\overline{n V_{E}^{\prime \prime 2}} / 2$. The analysis in Ref. 8 reveals that in this $k_{\perp}$ equation, the dominant source is the interchange term, while the dominant sink is the sheath loss term. A smaller role is played by the viscous term. Other contributions, including the transport term on the left-hand side of the equation, are negligible. 
To close the terms in this analytical $k_{\perp}$ equation, the following $1 \mathrm{D}$ mean-field transport model was proposed in Ref. 8:

$$
\begin{array}{rlrl}
\frac{\partial \bar{n}}{\partial t}+\nabla & \cdot\left(\bar{n} \bar{V}_{E}+\bar{\Gamma}_{E, t}-D_{n} \nabla_{\perp} \bar{n}\right)=-\sigma c_{s} \bar{n} \\
\bar{\Gamma}_{E, t} & =-C_{D} \sqrt{k_{\perp}} \nabla_{\perp} \bar{n}, & & C_{D}=23.9, \\
g T \bar{\Gamma}_{E, t} & =C_{\operatorname{sink}} \sqrt{\sigma} c_{s} \bar{n} k_{\perp}, & & C_{\text {sink }}=0.561,
\end{array}
$$

In these equations, $\bar{\Gamma}_{E, t}$ is the time-averaged particle flux due to ExB fluctuations. In the considered $1 \mathrm{D}$ case, the contribution from mean-flow phenomena to the total particle flux is negligible since the radial meanflow ExB velocity $\bar{V}_{E}$ is zero while $D_{n}$ is chosen to be small. Expression 7 for the turbulent particle flux follows from a regression analysis on TOKAM2D reference simulations, while equation 8 is obtained by assuming a local balance between the interchange source and sheath loss sink in equation 4 and filling out an analytical relation for the interchange source and a regression model for the sheath loss sink. The expression for the interchange source, $g T \bar{\Gamma}_{E, t}$, is analytically exact, while the sheath sink follows again from regression analysis. This one-equation $k_{\perp}$ model performs rather well over a range of TOKAM2D parameters. However, it is unable to capture trends for varying TOKAM2D viscosity $\nu$. This is not surprising as the viscous source in the $k_{\perp}$ equation has been crudely aggregated in the single sink term which predominantly models the sheath loss of $k_{\perp}$. Dimensionalising the diffusion coefficient in equation 7 yields $D \sim \rho_{\text {ref }} \sqrt{k_{\perp}}$. The global reference quantity $\rho_{\text {ref }}$ seems unnatural in this expression, as a $k(-\epsilon)$ model implies that turbulent transport can be described by local turbulent characteristics and does not depend on global reference quantities such as $\rho_{\text {ref }}$.

\section{Enstrophy equation and enstrophy bal- ance}

From hydrodynamic turbulence, it is known that viscous dissipation of kinetic energy is closely related to the enstrophy. Moreover, in hydrodynamic, inviscid, 2D turbulence, both the kinetic energy and the enstrophy are conserved. While the kinetic energy follows an inverse cascade, transferring energy from smaller to larger scales, the enstrophy follows a direct cascade $[2,15,16]$. Similar characteristics are expected for ExB drift turbulence $[2,17]$. Also, Tran et al.[18] have already shown that the enstrophy is related to zonal flow formation in $\mathrm{ExB}$ drift turbulence. Hence, it is expected that the turbulent enstrophy will provide valuable additional information not contained in the turbulent kinetic energy. Therefore, we define the total, mean flow and turbulent enstrophy as

$$
\bar{n} \zeta_{\text {tot }}=\frac{\overline{n \omega^{2}}}{2}, \quad \bar{n} \zeta_{\text {mean }}=\frac{\bar{n} \tilde{\omega}^{2}}{2}, \quad \bar{n} \zeta_{\perp}=\frac{\overline{n \omega^{\prime \prime 2}}}{2} .
$$

From the turbulent kinetic energy and the turbulent enstrophy, a characteristic time and length scale can be constructed. Using such dimensional combinations, the turbulent diffusion coefficient is expected to scale as 


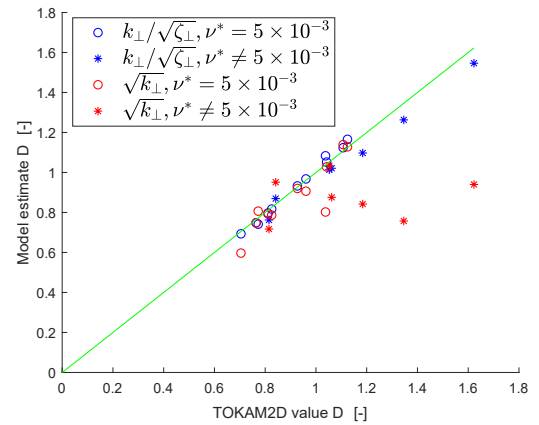

Figure 1: Scatter plot of diffusive relations.

$D \sim k_{\perp} / \sqrt{\zeta_{\perp}}$. Note that the one-equation $k_{\perp}$ model could only construct a velocity scale for the turbulence as only the turbulent kinetic energy was available. As a result, no dimensionally correct scaling for the diffusion coefficient could be constructed with turbulent characteristics only (as shown in equation 7).

Figure 1 shows a scatter plot comparing both diffusive relations. Each data point in the figure represents a TOKAM2D simulation for a certain set of parameters of which the data are averaged in time and in the diamagnetic and radial directions. Results of 18 simulations are used in which $g, \sigma$ and $\nu$ are respectively varied by a factor $0.5-1.5,0.3-1.5$ and 0.4-4 compared to the values used in Ref. 12 , while $T_{i}$ is varied between 0.3 and 4 (see appendix for exact simulation parameters). The horizontal axis shows the diffusion coefficient calculated from the TOKAM2D data directly, while the vertical axis shows an estimates for the diffusion coefficient obtained using the new model proposed here and the $k_{\perp}$ only model from equation 7. Both diffusive models are evaluated using the exact TOKAM2D values for $k_{\perp}$ and $\zeta_{\perp}$. While some scatter around the perfect fit line still remains, it is clear that the $k_{\perp}-\zeta_{\perp}$ estimate for the diffusion coefficient performs significantly better than the $k_{\perp}$ only estimate. In particular, the new model manages to capture trends with varying viscosity much better, confirming the hypothesis that the enstrophy is related to the viscous dissipation.

Since the previous has clearly shown the turbulent enstrophy to be an interesting quantity for modeling the average particle flux, which is ultimately the term that needs to be closed, it is worthwhile to derive an equation for the turbulent enstrophy. We follow a procedure similar to the work of Tran et al.[18], but allow independent density fluctuations, and we split the resulting equation into mean flow and turbulent contributions. Equations for the enstrophy can be derived analytically starting from TOKAM2D vorticity equation 2 . Multiplying this vorticity equation by $\omega$ yields an equation for the time rate of change of $\omega^{2}$. By multiplying with the density and using continuity equation 1 , an equation for $n \omega^{2}$ is found. Finally, averaging this equation over time yields an equation for the total enstrophy. Using similar techniques, an equation for the mean 
flow enstrophy is obtained by multiplying the vorticity equation with $n$, averaging over time, and multiplying the resulting equation with $\tilde{\omega}$. The difference between the total and the mean flow enstrophy equations then yields the turbulent enstrophy equation:

$$
\begin{array}{r}
\frac{\partial \bar{n} \zeta_{\perp}}{\partial t}+\nabla \cdot\left(\bar{n} \zeta_{\perp} \tilde{V}_{E}+\frac{\overline{n \omega^{\prime \prime 2} V_{E}^{\prime \prime}}}{2}\right)+\sigma c_{s} \frac{\overline{n \omega^{\prime \prime 2}}}{2} \exp \left(\Lambda^{*}-\frac{\phi}{T_{e}}\right) \\
=\underbrace{\overline{\omega^{\prime \prime}[p, g x]}}_{\text {interchange }}+\underbrace{\sigma \frac{c_{s} n \omega^{\prime \prime}\left(1-\exp \left(\Lambda-\frac{\phi}{T_{e}}\right)\right)}{\sigma c_{s}}}_{\text {sheath }}+\underbrace{\overline{n \omega^{\prime \prime} \nabla_{\perp}^{2} \omega}}_{\text {viscosity }} \\
-\overline{n \omega^{\prime \prime} V_{E}^{\prime \prime}} \cdot \nabla \tilde{\omega}+\frac{\overline{\omega^{\prime \prime 2} S_{n}}}{2}+D_{n} \frac{\overline{\omega^{\prime \prime 2} \nabla_{\perp}^{2} n}}{2} .
\end{array}
$$

As this equation has been derived analytically for the TOKAM2D model, it can be evaluated exactly using TOKAM2D data. A balance of the different terms in this equation is shown in figure 2 , together with a balance of the $k_{\perp}$ equation 4 . This figure clearly shows that the source of turbulent enstrophy, like that of turbulent kinetic energy, is dominated by the interchange term. However, sheath losses, viscosity and the subgrid dissipation seem to contribute almost equally to enstrophy losses, while the sink of the turbulent kinetic energy is dominated by the sheath losses only. Thus, these balances show that, as expected, $k_{\perp}$ and $\zeta_{\perp}$ have different characteristics. The subgrid scale term is a numerical imbalance in
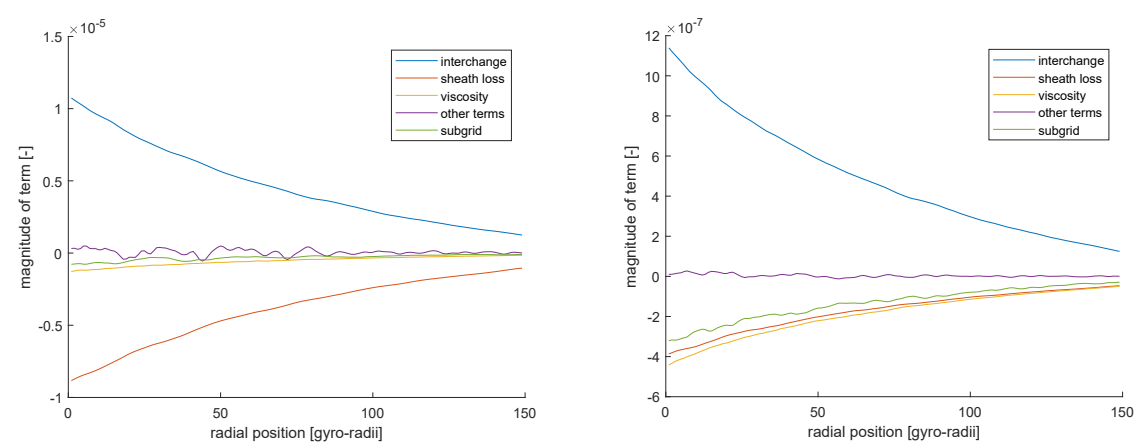

Figure 2: Balance of $k_{\perp}$ (left) and $\zeta_{\perp}$ (right) .

the evaluation of the different terms in the corresponding equations, and is caused by numerical dissipation as a result of the second order WENO schemes $[19,20]$ that are used in TOKAM2D [8]. This was confirmed by a grid refinement study: relative errors on both the $k_{\perp}$ and $\zeta_{\perp}$ equations decrease as the grid and the time step are refined. Simultaneously, $k_{\perp}$ and $\zeta_{\perp}$ increase, as their respective sinks decrease in magnitude. The larger error on the $\zeta_{\perp}$ equation compared to the one on the $k_{\perp}$ equation is most likely a result of the enstrophy being concentrated on the smaller scales which suffer more from the discretization, while the kinetic energy is more strongly present on larger scales. This difference in length scale 
is probably the reason why viscous dissipation is much more important for the $\zeta_{\perp}$ than it is for $k_{\perp}$. These explanations are in accordance with the understanding of hydrodynamic $2 \mathrm{D}$ turbulence and its dual cascade picture $[2,15,16]$.

\section{New two-equation $k_{\perp}-\zeta_{\perp}$ model}

This section proposes a model for the enstrophy equation derived in the previous section and integrates it in the mean-field transport model, together with the improved diffusive relation found in the previous section. The resulting two-equation $k_{\perp}-\zeta_{\perp}$ transport model proposed in this contribution is then

$$
\begin{gathered}
\frac{\partial \bar{n}}{\partial t}+\nabla \cdot\left(\bar{n} \bar{V}_{E}+\bar{\Gamma}_{E, t}-D_{n} \nabla_{\perp} \bar{n}\right)=-\sigma c_{s} \bar{n}, \\
\bar{\Gamma}_{E, t}=-C_{D} \frac{k_{\perp}}{\sqrt{\zeta_{\perp}}} \nabla_{\perp} \bar{n}, \\
g T \bar{\Gamma}_{E, t}=\frac{C_{\sigma, k} \sigma c_{s}}{T_{e}^{0.75} \frac{\bar{n} k_{\perp}^{2}}{\zeta_{\perp}}+C_{\nu, k} \nu n \zeta_{\perp},} \\
C_{\text {inter }, \zeta} g T \bar{\Gamma}_{E, t} \frac{\zeta_{\perp}}{k_{\perp}}=\frac{C_{\sigma, \zeta} \sigma c_{s}}{T_{e}} \bar{n} k_{\perp}+C_{\nu, \zeta} \nu \bar{n} \frac{\zeta_{\perp}^{2}}{k_{\perp}}, \\
C_{D}=7.71, \quad C_{\sigma, k}=4.43, \quad C_{\nu, k}=1.85, \\
C_{\text {inter }, \zeta}=0.974, \quad C_{\sigma, \zeta}=2.02, \quad C_{\nu, \zeta}=5.51 .
\end{gathered}
$$

The continuity equation 11 is the same as equation 6 from the oneequation $k_{\perp}$ model. The particle flux 12 is based on the diffusion coefficient proposed in section 3 . Equation 13 for $k_{\perp}$ retains the analytically exact relation for the interchange source from the the one-equation $k_{\perp}$ model i.e. $g T \bar{\Gamma}_{E, t}$, while the sinks on the right hand side are now split into a sheath loss and a viscous contribution. Equation 14 for $\zeta_{\perp}$ features terms representing the same three effects: the interchange source, sheath losses and viscous dissipation. Other terms (including transport) have been neglected in the $k_{\perp}$ and $\zeta_{\perp}$ equations at present as they appear to be small. All the model constants shown in 15 have been determined by means of term-by-term nonlinear least-squares regression using as reference data the results from the TOKAM2D simulations in appendix that are averaged in time and in the diamagnetic direction.

Algebraic expansion of the viscous dissipation term for the total kinetic energy shows that it scales as $-2 \nu \bar{n} \zeta_{\text {tot }}{ }^{1}$, which is in line with the findings of hydrodynamic $2 \mathrm{D}$ turbulence $[2,15,16]$. Hence, it seems reasonable to model the viscous dissipation of the turbulent kinetic energy based on a similar relation, but using the turbulent enstrophy instead of the total enstrophy. This also explains that $C_{\nu, k}$ is approximately but not exactly equal to two. A similar expansion, again in agreement with hydrodynamic $2 \mathrm{D}$ turbulence $[2,15,16]$, shows that the viscous dissipation of total enstrophy scales as $-2 \nu \bar{n} P$, where $\bar{n} P=\overline{n\left(\nabla_{\perp}^{2} \omega\right)^{2}} / 2$ is the palinstrophy. The viscous dissipation of turbulent enstrophy is therefore modeled using a dimensionally correct combination for the (turbulent)

\footnotetext{
${ }^{1}$ In this scaling and the next ones in this paragraph some additional terms have been neglected, since TOKAM2D evaluation showed them to be negligible.
} 
palinstrophy $P \sim \zeta_{\perp}^{2} / k_{\perp}$. A series expansion in $\phi^{\prime \prime}$ shows that the sheath loss of the turbulent enstrophy is proportional to $-2 \sigma c_{s} \bar{n} k_{\perp}$. Thus, four of the six terms in the $k_{\perp}-\zeta_{\perp}$ system are modeled based on physical and mathematical insights. The remaining terms, the sheath loss of $k_{\perp}$ and interchange source of $\zeta_{\perp}$, have been modeled purely based on regression analysis of TOKAM2D data. While the models for both terms manage to fit the data sufficiently well, it is not clear if they really capture the underlying physics. In particular, the model for the interchange source of $\zeta_{\perp}$ requires further investigation, as it is a dominant term in the equation. Hence, improvements in the model for this term and the sheath loss term for $k_{\perp}$ are expected to further enhance the performance of the transport model presented in this paper.

We remark that both the one-equation and two-equation models imply the same turbulence saturation mechanism, similar in nature to gradient removal assumptions [21]. The turbulent ExB particle flux (and hence density (pressure) gradient) appears directly in the source terms of the equations for $k_{\perp}$ and $\zeta_{\perp}$. Too steep gradients will lead to an increase in the turbulence intensity and resulting turbulent transport, tending to flatten out the gradients until a balance between particle flux and turbulence intensity is found.

An interesting feature of the equation set above is that $k_{\perp}$ and $\zeta_{\perp}$ equations 13 and 14 differ only by a factor $\zeta_{\perp} / k_{\perp}$ and a number of constants. This allows both equations to be written and solved together as a linear matrix system:

$$
\left[\begin{array}{c}
g T \bar{\Gamma}_{E, t} \\
C_{\text {inter }, \zeta} g T \bar{\Gamma}_{E, t}
\end{array}\right]=\left[\begin{array}{cc}
\frac{C_{\sigma, k} \sigma c_{s}}{T_{e}^{0.75}} & C_{\nu, k} \nu \\
\frac{C_{\sigma, \zeta}^{0} \sigma c_{s}}{T_{e}} & C_{\nu, \zeta} \nu
\end{array}\right]\left[\begin{array}{c}
\bar{n} \frac{k_{\perp}^{2}}{\zeta} \\
\bar{n} \zeta
\end{array}\right]
$$

It can be noted that the two-equation $k_{\perp}-\zeta_{\perp}$ approach to modeling turbulence bears a lot of similarity to $k-\epsilon$ models that are commonly used to model hydrodynamic turbulence in a Reynolds-Averaged Navier-Stokes (RANS) approach $[7,13]$. There as well, the combination of both quantities allows to construct a length and a time scale for the turbulence and sources and sinks of $k$ and $\epsilon$ differ by a factor $\epsilon / k$. However, in the model presented here, both equations represent the evolution of a turbulent quantity and all of the terms in both equations have a clear physical meaning, while in RANS modelling of hydrodynamic turbulence $\epsilon$ is typically an ad-hoc quantity used to represent dissipation on the small scales.

\section{Performance of the two-equation $k_{\perp}-\zeta_{\perp}$ model}

The left and middle figures of figure 3 show scatter plots of the observed TOKAM2D values for $k_{\perp}$ and $\zeta_{\perp}$ versus the estimate for these quantities obtained with the two-equation $k_{\perp}-\zeta_{\perp}$ model and the one-equation $k_{\perp}$ model. The former estimate is obtained by solving system 16 for $k_{\perp}$ and $\zeta_{\perp}$ with all other quantities filled out using exact TOKAM2D data. Additionally, the TOKAM2D results for the subgrid model have been added to the left hand side of system 16 to cope with the large error on the 
enstrophy equation especially. The $k_{\perp}$ estimate for the one-equation $k_{\perp}$ model is likewise obtained from equation 8. However, no correction for the subgrid model is made in this case as this error is relatively small for the $k_{\perp}$ equation and because this subgrid dissipation is implicitly present in the sink constant of equation 8 . Hence, figure 3 shows that the newly developed model manages to capture the trends in the TOKAM2D parameter space very well, both for $k_{\perp}$ and $\zeta_{\perp}$. While some scatter around the perfect fit line does remain, this error is significantly smaller than that for the one equation $k_{\perp}$ model.
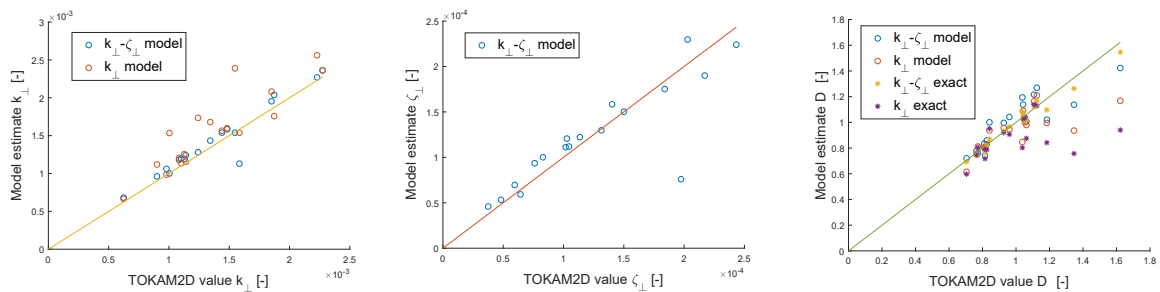

Figure 3: Scatter plot of $k_{\perp}$ (left), $\zeta_{\perp}$ (middle) and $D$ (right) estimates for the $k_{\perp}-\zeta_{\perp}$ model and the $k_{\perp}$ model .

The right figure in figure 3 shows a similar scatter plot for the diffusion coefficient. The points indicated by circles are obtained by filling out the model values for $k_{\perp}$ and $\zeta_{\perp}$ in particle transport relations 12 and 7 respectively. The points indicated by asterisks are obtained by evaluating these diffusion coefficients using exact TOKAM2D values for $k_{\perp}$ and $\zeta_{\perp}$ (thus, same data points as shown in figure 1). The right figure in figure 3 shows that the estimate of the diffusion coefficient of the two-equation $k_{\perp}-\zeta_{\perp}$ model is not unequivocally superior to that of the one-equation $k_{\perp}$ model. While the former seems to better capture the trend of the high $D$ simulations, it is still far from perfect. For the simulations at intermediate levels of $D$, the new model seems to perform slightly worse than the original one-equation model. This is rather surprising as it has clearly been shown that the diffusion relation is more reliable, that the estimate for $k_{\perp}$ is more accurate, and that the estimate for $\zeta_{\perp}$ looks very reasonable. It seems that the remaining errors on $k_{\perp}$ and $\zeta_{\perp}$ reinforce each other in the diffusive relation and cause the overall behavior to become less accurate. Further research is needed to determine how the accuracy depends on the value of the model constants and on the possibly lacking models for the interchange source of $\zeta_{\perp}$ and sheath loss sink of $k_{\perp}$.

Figure 4 shows the radial profiles of $k_{\perp}, \zeta_{\perp}$ and $D$ for the TOKAM2D simulation with the default parameter setting (see appendix for exact parameters). The figure shows that both models manage to capture the trends in the radial profiles rather well.

\section{Conclusion}

In this contribution, a two-equation $k_{\perp}-\zeta_{\perp}$ model has been presented to approximately model the mean-field transport due to ExB drift turbu- 

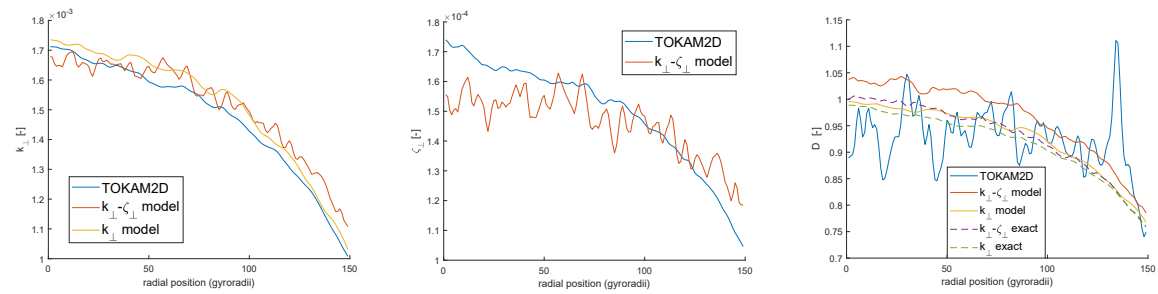

Figure 4: Radial profile of $k_{\perp}$ (left), $\zeta_{\perp}$ (middle) and $D$ (right) estimates for the $k_{\perp-} \zeta_{\perp}$ model and the $k_{\perp}$ model .

lence in the SOL. The model extends the one-equation $k_{\perp}$ model presented by Coosemans et al.[8]. The turbulent enstrophy, a new quantity further characterizing the intensity of the turbulence has been added to the equation set and has been modeled rather satisfactorily. Combining the turbulent kinetic energy and enstrophy allows to construct time and length scales for the turbulence, which has yielded an improved model for the turbulent diffusion coefficient. In particular, the new model is able to capture more accurately the scaling of the diffusion coefficient with the viscosity.

This work can form the basis for improved transport descriptions in 2D mean-field modeling. A first version of such improved transport model has been implemented in SOLPS-ITER by Carli et al.[22].

It is expected that future research on the sheath loss term for $k_{\perp}$ and interchange term for $\zeta_{\perp}$, which are now modeled empirically, will further improve the model presented here. Extension of the present model to nonisothermal plasmas is also envisaged in the future, as well as modeling the edge plasma inside the last closed flux surface.

\section{Acknowledgments}

The first author wishes to acknowledge fruitful discussion with T.T. Tran, which triggered the idea of including the turbulent enstrophy in our transport model.

R. Coosemans is funded by a strategic basic research grant of the Research Foundation Flanders (FWO), file number 48697. The computational resources and services used in this work were provided by the VSC (Flemish Supercomputer Center), funded by the Research Foundation Flanders (FWO) and the Flemish Government - department EWI.

\section{TOKAM2D Simulation parameters}

In all simulations the parameters were chosen such that $D_{n}=\nu, \sigma_{N}=$ $\sigma_{W}, \Lambda=2.8388$ and $T e 0=1$. All reported simulations were run for the isothermal version of the code with a non-periodic $\mathrm{x}$-direction and using the strong Boussinesq assumption. The default settings for the simulations are shown in table 1. 
Table 1: Default parameters used in TOKAM2D simulations and their postprocessing.

\begin{tabular}{l|l|l|l|l|l|l|l|l|l|l}
$\mathrm{Lx}$ & $\mathrm{Ly}$ & $t_{\text {start }}$ & $t_{\text {end }}$ & $d x$ & $d y$ & $d t$ & $g$ & Ti0 & $\sigma$ & $\nu$ \\
\hline 256 & 256 & $2 \mathrm{e} 5$ & $8 \mathrm{e} 5$ & 1 & 1 & 1 & $6 \mathrm{e}-4$ & 1 & $1 \mathrm{e}-4$ & $5 \mathrm{e}-3$
\end{tabular}

Table 2 shows the parameters of the simulations used for the regression analysis. Only the parameters that differ from the default simulation (for which the parameters are listed in table 1) are shown, except for the third simulation which is the default simulation.

Table 2: TOKAM2D and post-processing parameters of the simulations used in the regression analysis.

\begin{tabular}{l|l|l|l|ll|l|l|l|l} 
Nr. & $g$ & Ti & $\sigma$ & $\nu$ & Nr. & $g$ & $T i 0$ & $\sigma$ & $\nu$ \\
\hline 1 & $4 \mathrm{e}-4$ & & & & 10 & $9 \mathrm{e}-4$ & & $5 \mathrm{e}-5$ & $2 \mathrm{e}-2$ \\
\hline 2 & $4.5 \mathrm{e}-4$ & & & & 11 & & & $8 \mathrm{e}-5$ & \\
\hline 3 & $6 \mathrm{e}-4$ & 1 & $1 \mathrm{e}-4$ & $5 \mathrm{e}-3$ & 12 & $4.5 \mathrm{e}-4$ & & $1.5 \mathrm{e}-4$ & \\
\hline 4 & $7.5 \mathrm{e}-4$ & & & & 13 & & 0.5 & & \\
\hline 5 & $9 \mathrm{e}-4$ & & & & 14 & & 2 & & \\
\hline 6 & & & & $2 \mathrm{e}-3$ & 15 & $3 \mathrm{e}-4$ & 4 & & $7 \mathrm{e}-3$ \\
\hline 7 & & & & $1 \mathrm{e}-2$ & 16 & $7 \mathrm{e}-4$ & 0.7 & $3 \mathrm{e}-5$ & \\
\hline 8 & & & & $1.5 \mathrm{e}-2$ & 17 & & 0.3 & $1.5 \mathrm{e}-4$ & $8 \mathrm{e}-3$ \\
\hline 9 & & & $5 \mathrm{e}-5$ & $2 \mathrm{e}-2$ & 18 & $4 \mathrm{e}-4$ & 0.4 & $6 \mathrm{e}-5$ &
\end{tabular}

\section{References}

[1] J. Wesson. Tokamaks 3rd ed. Oxford: Clarendon Press, Oxford, 2004.

[2] W. Fundamenski. Power Exhaust In Fusion Plasmas. Cambridge University Press, Cambridge, 2010.

[3] B. D. Scott. The character of transport caused by ExB drift turbulence. Phys. Plasmas, 10(4):963, 2003.

[4] L. Aho-Mantila, M. Wischmeier, H.W. Müller, et al. Outer divertor of ASDEX Upgrade in low-density L-mode discharges in forward and reversed magnetic field: I. comparison between measured plasma conditions and SOLPS5.0 code calculations. Nucl. Fusion, 52(10):103006, 2012 .

[5] F. Reimold, M. Wischmeier, M. Bernert, et al. Experimental studies and modeling of complete H-mode divertor detachment in ASDEX upgrade. J. Nucl. Mater., 463:128-134, 2015. 
[6] W. Dekeyser, X. Bonnin, S. W. Lisgo, et al. SOLPS-ITER modeling of the Alcator C-Mod divertor plasma. Plasma and Fusion Res., 11:1403103, 2016.

[7] H. Bufferand, G. Ciraolo, Ph. Ghendrih, et al. Interchange turbulence model for the edge plasma in SOLEDGE2D-EIRENE. Contrib. Plasma Phys., 56(6-8):555 - 562, 2016.

[8] R. Coosemans, W. Dekeyser, and M. Baelmans. Turbulent kinetic energy in tokam2d: $k_{\perp}$ balance and relation to particle transport. 2019. https://arxiv.org/abs/1912.04346v1 arXiv:1912.04346v1 [physics.plasm-ph], to be submitted.

[9] S. Baschetti, H. Bufferand, G. Ciraolo, et al. A $k$ - $\epsilon$ model for plasma anomalous transport in tokamaks: closure via the scaling of the global confinement. Nuclear Materials and Energy, 19:200-204, 2019.

[10] Y. Sarazin and Ph. Ghendrih. Intermittent particle transport in twodimensional edge turbulence. Phys. Plasmas, 5(12):4214-4228, 1998.

[11] N. Nace. Dynamics of driven and spontaneous transport barriers in the edge plasma of tokamaks. PhD thesis, Aix-Marseille University, 2018. available at http://www.theses.fr/2018AIXM0101.

[12] Y. Marandet, N. Nace, M. Valentinuzzi, et al. Assessment of the effects of scrape-off layer fluctuations on first wall sputtering with the tokam-2d turbulence code. Plasma Phys. Control. Fusion, 58(11):114001, 2016.

[13] S. B. Pope. Turbulent Flows. Cambridge University Press, Cambridge, 2015.

[14] V. M. Canuto. Compressible turbulence. Astrophys. J., 482:827-851, 1997.

[15] A. Alexakis and C.R. Doering. Energy and enstrophy dissipation in steady state 2d turbulence. Phys. Lett., 359(6):652-657, 2006.

[16] V. Yakhot. Universal law of enstrophy decay in two-dimensional large-reynolds-number turbulence. Phys. Rev. Lett., 93(1):014502, 2004 .

[17] S.J. Camargo, D. Biskamp, and B. D. Scott. Resistive drift-wave turbulence. Phys. Plasmas, 2(1):48-62, 1995.

[18] T.T. Tran, S.S. Kim, H. Jhang, et al. Turbulence characteristics, energy equipartition, and zonal flow generation in coupled drift waveparallel velocity gradient driven turbulence. Plasma Phys. Contr. Fusion, 61(6):065002, 2019.

[19] G.-S. Jiang and C.-W. Shu. Efficient implementation of weighted eno schemes. J. Comput. Phys, 126(0130):202-228, 1996. 
[20] M.P. Martín, E.M. Taylor, M. Wu, et al. A bandwidth-optimized weno scheme for the effective direct numerical simulation of compressible turbulence. J. Comput. Phys, 220:270-289, 2006.

[21] P. Ricci and B. N. Rogers. Plasma turbulence in the scrape-off layer of tokamak devices. Phys. Plasmas, 20:010702, 2013.

[22] S. Carli, W. Dekeyser, R. Coosemans, et al. Turbulence-based radial transport models for solps-iter: a compass case study. These proceedings, 2019. 